\title{
Floristic Diversity Assessment of Home Garden in Palayamkottai Region of Tirunelveli District, Tamil Nadu a Means of Sustainable Biodiversity Conservation
}

\author{
J. Vijayakumari, V. Sundara Prabha, E. Jebarubi, T. Leon Stephan Raj, S. Beschi Antony Rayan \\ Post Graduate and Research Department of Botany, \\ St. Xavier's College (Autonomous), Palayamkottai, Tamil Nadu, India
}

\begin{abstract}
How to cite this paper: J. Vijayakumari | V.Sundara Prabha| E. Jebarubi| T. Leon Stephan Raj | S. Beschi Antony Rayan "Floristic Diversity Assessment of Home Garden in Palayamkottai Region of Tirunelveli District, Tamil Nadu a Means of Sustainable Biodiversity Conservation" Published in International Journal of Trend in Scientific Research and Development (ijtsrd), ISSN: 24566470, Volume-3 | Issue-3, April 2019, pp.1484-1491, URL: https://www.ijtsrd.c om/papers/ijtsrd23 390.pdf

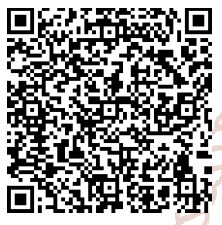

IITSRD23390

Copyright (C) 2019 by author(s) and International Journal of Trend in Scientific Research and Development Journal. This is an Open Access article distributed under the terms of the Creative Commons

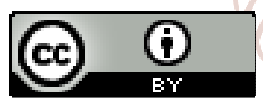
Attribution License (CC BY 4.0) (http://creativecommons.org/licenses/ by/4.0)
\end{abstract}

\section{ABSTRACT}

Home garden is an integrated system which consists of different plants in its small area that produces a variety of foods and agricultural products including vegetables, fruits, medicinal plants, ornamentals etc. All home gardeners were individually interviewed about home garden management and plant utilization, among other information. The biological and cultural significance of agro biodiversity in home gardens are highlighted with the future obligation and prospect in home gardens to improve our natural resource and avoid the pollution with free air circulation. The main objective of this study was to assess the status, composition and diversity of plants in an urban home gardens with the help of socio economic factors of households. The study was carried out in Palayamkottai region of Tirunelveli district of Tamil Nadu, India. A total of 182 species were observed, which belonging to 159 genera and 71 families. Most of plants were dicot with 53 families and 150 species, whereas monocot with 15 families and 29 species and third one gymnosperm were presented with 2 families and 2 species and a single species of Pteridophyte also were documented. Fabaceae, Apocyanaceae and Solanaceae were the most dominant family in this home garden.

KEYWORDS: Home garden, biodiversity, agricultural, medicinal plant and ornamental

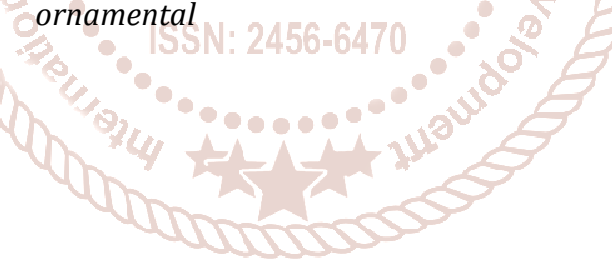

\section{INTRODUCTION}

Home gardens are traditional agro forestry systems characterized by the intricacy of their structure with lot of functions. Home gardens can be defined as 'land use system involving deliberate management of multipurpose biological varieties such as trees, herbs and shrubs in intimate association with annual and perennial agricultural crops and variable livestock within the compounds of individual houses ${ }^{1}$. Home garden is an integrated system which comprises different things in its small area (the family house, a kitchen garden, a mixed garden etc). It produces a variety of foods, medicine and agricultural products. These products could be used both for home consumption and for incoming purpose. There are numerous types of home gardens were serving for several functions. Vegetable gardens were the cultivation of different kinds of vegetables and fruits. Herbal gardens can be grown in a small a spot as a window box. Growing of culinary herbs and spices gives the freshest ingredients. Different herbs are usually separated through the planting design and cultivated in pot. Rose gardens mainly for the cultivation of flowers for income or beautification and fourth one knot gardens had squares of flora or paving encased by dwarf. The knot garden looks to control nature to into beautiful patterns. Oriental gardens are incorporates the use of water and paths, rocks and sculptured bushes and trees to create a peaceful and tranquil garden. Wild garden is a natural type of garden. Its natural style encourages the growth of natural plants and wild grasses. Home gardens are cultivation systems for both food and non-food production. Nevertheless, home gardens are mostly known for their food production function considered to be their basic function ${ }^{2}$. The different denominations associated to home gardens are home food gardens, urban food gardens, domestic food gardens and kitchen garden $3,4 \& 5$ are evidences of the paramount importance attributed to food production function of home gardens in the available literature. However, based on the spectrum of home gardens eco-friendly services $6,7,8,9 \& 10$ and the different uses reported to be combine to home gardens ${ }^{11,12 \& 13}$, the non-food productions (medicinal, ornamental, etc.) are also of importance especially in some topographical framework. For 
instance in Benin, where the reported plant used for nonfood purposes compare to food ones ${ }^{12}$ it should be expected that home gardens are functionally diverse. Because food and health care are basic human needs, we predict that food and medicinal function will predominate other functions. Home garden species typically have multiple uses, meeting family needs for food, medicine, shade, religious rituals and ornamental purposes ${ }^{14}$, and these species can be cultivated, tolerated, enhanced, and protected ${ }^{15}$.Tolerance includes practices within human-made environments that are directly related to the conservation of useful plants that existed before the environments were transformed. Currently, the floristic composition of home gardens tends toward ornamental plants ${ }^{16}$.Not all plants receive the same attention. The choice of certain species is associated with their use in community celebrations or as foods consumed in everyday life and the use of certain species to treat diseases for generations ${ }^{17}$.A clear differentiation of the floristic composition between Mestizo and Maya home gardens ${ }^{18}$.In
Latin America, the purpose of home gardens is related to the edible, medicinal and aesthetic uses of a particular species, which are the most common factors due to the high frequency of these uses ${ }^{19}$. In this study, the floristic composition of home gardens, the contribution of edible and medicinal species to household subsistence in Palayamkottai region of Tirunelveli District, Tamil Nadu, India.

\section{MATERIALS AND METHODS \\ Study Area}

The present study was conducted in Palayamkottai (8.7166 음 $\mathrm{N}$ and $\left.77.7333^{\circ} \mathrm{E}\right)$ region of Tirunelveli district of Tamil Nadu, India. Temperature 28 ${ }^{\circ} \mathrm{C}$ and humidity $78 \%$. Palayamkottai was called as the Oxford of the southern Tamil Nadu. It is around $189.9 \mathrm{~km}^{2}$ and the population about 4, 73,637. Mainly four areas were considered in this study such as KTC Nagar, Perumalpuram, Santhi Nagar and NGO Colony (Fig.1).

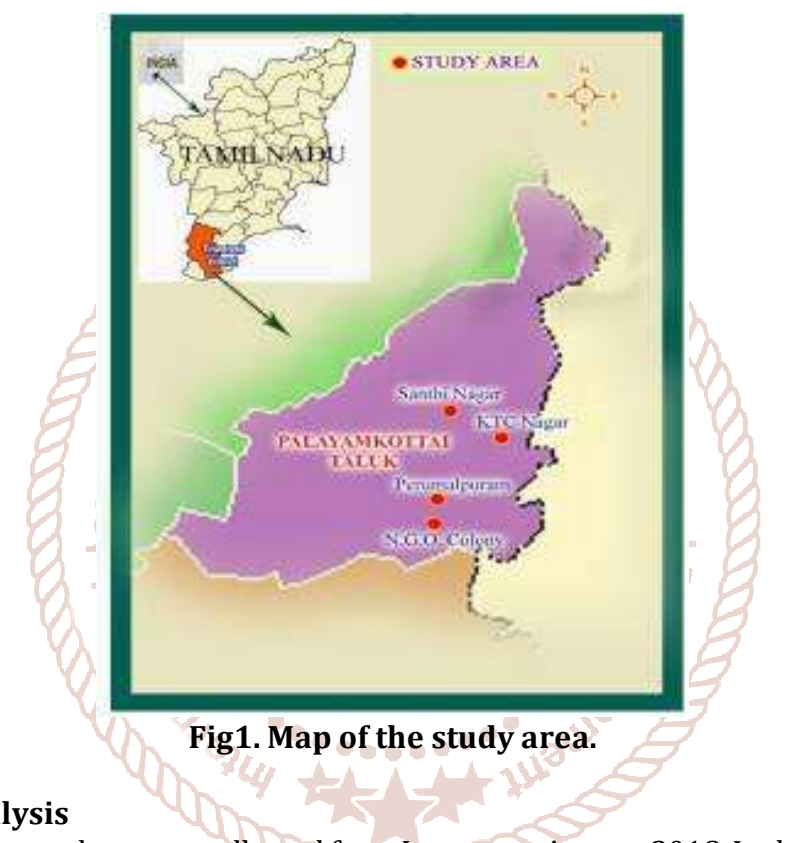

Field information from these home gardens was collected from January to August, 2018. In the first interview, each interviewee was asked the following questions to obtain a list of the most frequently mentioned home garden plants. For this analysis 182 species were considered. The botanical material was identified with the support of college Herbarium for St. Xavier's college of Palayamkottai and experts of taxonomy. Households were identified as sampling units for the survey.

\section{RESULT AND DISCUSSION}

A total of 182 plant species were collected from the Palayamkottai region of Tirunelveli District. In the Habitat, most of the home harden plants 32\% (60 species) were trees, $21 \%$ (39 species) were shrubs, 33\% (61 species) were herbs, $10 \%$ were (18 species) climbers and 4\% (7 species ) were twiners (Fig.2).

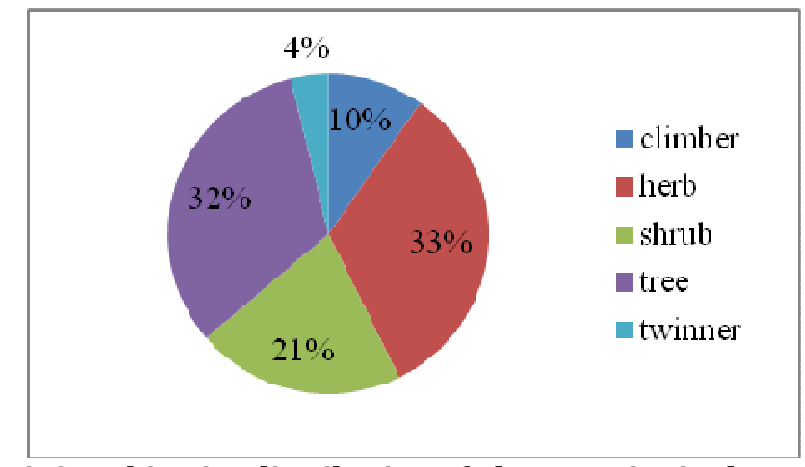

Fig2. Habit wise distribution of plant species in the study area.

The 182 plant species (including a Gymnosperm and Pteridophyte) belonging to 159 genera and 71 families. 
International Journal of Trend in Scientific Research and Development (IJTSRD) @ www.ijtsrd.com eISSN: 2456-6470

Table No. 1: List of plant species from Palayamkottai, Tirunelveli District

\begin{tabular}{|c|c|c|c|c|}
\hline $\begin{array}{l}\text { S. } \\
\text { No }\end{array}$ & Botanical Name & Vernacular Name & Family & Habit \\
\hline 1. & Andrographis paniculata(Burm.f.) Wall. ex Nees & Siriyanangai & Acanthaceae & Herb \\
\hline 2. & Barleria cristata L. & December poo & Acanthaceae & Herb \\
\hline 3. & Barleria prionitis L & Sulli flower & Acanthaceae & Herb \\
\hline 4. & Crossandra infundibuliformis (L.) Nees & Kanakambaram & Acanthaceae & Herb \\
\hline 5. & Justicia adhatoda L. & Aadathoda & Acanthaceae & Shrub \\
\hline 6. & $\begin{array}{l}\text { Pseudoranthemum laxiflorum (A. Gray). } \\
\text { F.T.Hubb.ex.L.H.Bailey }\end{array}$ & Nagamalli & Acanthaceae & Shrub \\
\hline 7. & Thunbergia grandiflora (Roxb. ex Rottl.) Roxb & -------------- & Acanthaceae & Climber \\
\hline 8. & Acorurs calamus L. & Vasambu & Acoraceae & Herb \\
\hline 9. & Alternanthera sessilis (L.) R. Br. ex DC. & Ponnankanni keerai & Amaranthaceae & Herb \\
\hline 10. & Amaranthus dubius Mart.exThell & Thandu keerai & Amaranthaceae & Herb \\
\hline 11. & Amaranthus viridis L. & Kuppai keerai & Amaranthaceae & Herb \\
\hline 12. & Celosia argentea L. & Kozhi kondai & Amaranthaceae & Herb \\
\hline 13. & Gomphrena globosa L. & Vaada malli & Amaranthaceae & Herb \\
\hline 14. & Achyranthus bidentata Blume & Sennaiyuruvi & Amaranthaceae & Herb \\
\hline 15. & Zephyranthes candida(Lindl). Herb. & Rain lily & Amaryillidaceae & Herb \\
\hline 16. & Polianthes tuberosa L. & Nila Sampangi & Amaryliceaeae & Herb \\
\hline 17. & Allium cepa L. & Vengayam & Amaryllidaceae & Herb \\
\hline 18. & Crinum asiaticum L. & Visha mungil & Amaryllidaceae & Herb \\
\hline 19. & Anacardium occidentale L. & Kollampalam & Anacardiaceae & Tree \\
\hline 20. & Mangifera indica L. & Maamaram & Anacardiaceae & Tree \\
\hline 21. & Annona squamosa L. & Seethapalam & Annonaceae & Tree \\
\hline 22. & Polyalthia longifolia Sonn. & Nettulingam & Annonaceae & Tree \\
\hline 23. & Centella asiatica (L.) Urban & Vallarai keerai & Apiaceae & Herb \\
\hline 24. & Coriandrum sativum L. & Malli elai & Apiaceae & Herb \\
\hline 25. & Allamanda cathartica L. $\quad 8$ वे & Allamanda poo & Apocyanaceae & $\begin{array}{l}\text { Hardy } \\
\text { climber }\end{array}$ \\
\hline 26. & Hemidemus indicus (L.) R.Br. & Nannaari & Apocyanaceae & Twiner \\
\hline 27. & Nerium oleander L. $\quad$ Of Trend In & Arali poo & Apocyanaceae & Shrub \\
\hline 28. & Plumeria alba L. & Nela Sampangi & Apocyanaceae & Tree \\
\hline 29. & Plumeria rubra $\mathrm{L}$. & $\cdots$ & Apocyanaceae & Tree \\
\hline 30. & $\begin{array}{l}\text { Adenium obesum (Forssk.) } \\
\text { Roem. \& Schult. }\end{array}$ & Desert rose & Apocynaceae & Shrub \\
\hline 31. & Calotropis gigantea (L.) Dryand. & Erukam poo & Apocynaceae & Shrub \\
\hline 32. & Cascabela thevetia (L.) Lippold & Ponnarali & Apocynaceae & Shrub \\
\hline 33. & Catharanthus roseus (L.) G. Don & Nithiya kalyani & Apocynaceae & Herb \\
\hline 34. & Plumeria pudica Jacq. & Perungalli & Apocynaceae & Shrub \\
\hline 35. & $\begin{array}{l}\text { Tabernaemontana divaricata (L.) R.Br.exRoem. \& } \\
\text { Schult. }\end{array}$ & Nanthiya vattam & Apocynaceae & Shrub \\
\hline 36. & Colacasia esculenta (L.) Schott & Seppa Kilangu & Araceae & Herb \\
\hline 37. & Monstera deliciosa Liebm. & Windowleaf & Araceae & Climber \\
\hline 38. & Araucaria columnaris J.R.Forst. Hook. & Christmas maram & Araucariaceae & Tree \\
\hline 39. & Cocos nucifera L. & Thennai & Arecaceae & Tree \\
\hline 40. & Dypsis lutescens (H.Wendl.) Beentje\&J.Dransf. & Areca palm & Arecaceae & Herb \\
\hline 41. & Epipremnum aureum (Linden \&Andre) G.S. Bunting & Money plant & Arecaceae & Climber \\
\hline 42. & Phoenix dactylifera L. & Perichai & Arecaceae & Tree \\
\hline 43. & Rhapis excelsa (Thunb.) A.Henry & Injambakkam & Arecaceae & Shrub \\
\hline 44. & Agave americana L. & Anaikathalai & Asparagaceae & Shrub \\
\hline 45. & Asparagus racemosus Willd. & Thanneer vittan & Asparagaceae & Climber \\
\hline 46. & Chlorophytum comosum (Thunb.) Jacques & --------- & Asparagaceae & Herb \\
\hline 47. & Draceaena reflexa Lam. & ---------- & Asparagaceae & Tree \\
\hline 48. & Sansevieria trifaciata Prain & Paambu kattralai & Asparagaceae & Herb \\
\hline 49. & Aloe vera (L.) Burm.f. & Kathalai & Asphodelaceae & Herb \\
\hline 50. & Chrysanthemum indicum L. & Saamanthi & Asteraceae & Herb \\
\hline 51. & Chrysogonum peruvianumL. & Zinnia poo & Asteraceae & Herb \\
\hline 52. & Eclipta prostrata (L.) L. & Karisilanganni & Asteraceae & Herb \\
\hline 53. & Helianthus annuus L. & Suriyagandhi poo & Asteraceae & Shrub \\
\hline 54. & Tagetes erecta L. & Tulukka malligai & Asteraceae & Herb \\
\hline 55. & Impatiens balsaminaL. & Kacittumpai & Balsaminaceae & Herb \\
\hline 56. & Basella alba L. & Pasalai keerai & Basellaceae & Twiner \\
\hline
\end{tabular}


International Journal of Trend in Scientific Research and Development (IJTSRD) @ www.ijtsrd.com eISSN: 2456-6470

\begin{tabular}{|c|c|c|c|c|}
\hline 57. & Tecoma stans (L.) Juss. ex Kunth & Nakacenpakam & Bignoniaceae & Shrub \\
\hline 58. & Cordia sebestena L. & Aechinaruvihli & Boraginaceae & Tree \\
\hline 59. & Brassica juncea (L.) Czern. & Kadugu & Brassicaceae & Herb \\
\hline 60. & Ananas cosmosus (L.) Merr & Annachi & Bromeliaceae & Shrub \\
\hline 61. & Mammillaria baumii Boed & ---------- & Cactaceae & Shrub \\
\hline 62. & Opuntia dillenii (Ker-Gawl.) Haw. & Sappathikalli & Cactaceae & Shrub \\
\hline 63. & Tamarindus indica L. & Pulia maram & Fabaceae & Tree \\
\hline 64. & Phanera purpurea (L.) Benth. & Nilattiruvatti & Fabaceae & Tree \\
\hline 65. & Carica papaya L. & Pappali & Caricaceae & Tree \\
\hline 66. & Casuarina equisetifolia L. & Savukku & Casuariniaceae & Tree \\
\hline 67. & Saraca asoca (Roxb.) Willd. & Asogamaram & Caesalpiniaceae & Tree \\
\hline 68. & Senna alexandrina Mill. & Alakalam & Caesalpiniaceae & Shrub \\
\hline 69. & Senna auriculata(L.) Roxb. & Aavaram poo & Caesalpiniaceae & Tree \\
\hline 70. & Combretum constrictum (Benth.) & Maruthamaram & Combretaceae & Tree \\
\hline 71. & Combretum indicum (L.)DeFilipps & Irangun malli & Combretaceae & Tree \\
\hline 72. & Tradescantia pallida (Rose) D.R.Hunt & Paccaialari & Commelinaceae & Herb \\
\hline 73. & Ipomoea quamoclit L. & Mayir manikkam & Convolvulaceae & Twiner \\
\hline 74. & $\begin{array}{l}\text { Chamaecostus cuspidatus } \\
\text { (Nees \& Mart.) C.Specht \& D.W.Stev. }\end{array}$ & Neyccarikamaram & Costaceae & Herb \\
\hline 75. & Costus woodnii L. & ------------ & Costaceae & Herb \\
\hline 76. & Costus igneus Nak & Insulin chedi & Costaceae & Herb \\
\hline 77. & Citrullus lanatus (Thunb.) Matsum. \& Nakai & Dharpoosani & Cucurbitaceae & Climber \\
\hline 78. & Coccinia grandis (L.) Voigt & Kovaka & Cucurbitaceae & Climber \\
\hline 79. & Cucumis sativusL. & Vellarikai & Cucurbitaceae & Climber \\
\hline 80. & Cucurbita maxima Duchesne & Poosanikai & Cucurbitaceae & Climber \\
\hline 81. & Lagenaria siceraria (Molina) Standl. & Suraikai & Cucurbitaceae & Climber \\
\hline 82. & Luffa acutangula Mill. & Peerkankai & Cucurbitaceae & Climber \\
\hline 83. & Momordica charantia L. & Pavaikai & Cucurbitaceae & Climber \\
\hline 84. & Cupressus sempervirens L. & Churam & Cupressaceae & Tree \\
\hline 85. & Cycas circinalis L. & Madanakama poo & Cycadaceae & Tree \\
\hline 86. & Cycas revolutaThunb. & 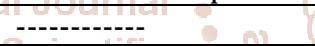 & Cycadaceae & Tree \\
\hline 87. & Muntingia calabura L. & Then pazham & Elaeocarpaceae & Tree \\
\hline 88. & Euphorbia cyatophora Murray & Thiththili poo & Euphorbiaceae & Herb \\
\hline 89. & Codiaeum variegatum (L.) Rumph. ex A.Juss Develo & Aathuppoondu & Euphorbiaceae & Shrub \\
\hline 90. & Euphorbia hirta L. & Amman pacharicy & Euphorbiaceae & Herb \\
\hline 91. & Euphorbia milii Des Moul. $\quad$ LSN: 24 & Kreeda kalli & Euphorbiaceae & Shrub \\
\hline 92. & Euphorbia tithymaloides L. & Kaṇnadi kalli & Euphorbiaceae & Shrub \\
\hline 93. & Euphorbia trigona Mill. & Paal kalli & Euphorbiaceae & Herb \\
\hline 94. & Ricinus communis L. & Amanakku & Euphorbiaceae & Shrub \\
\hline 95. & Arachis hypogaeaL. & Ver kadalai & Fabaceae & Herb \\
\hline 96. & Clitoria ternatea L. & Shangu pushpam & Fabaceae & Twiner \\
\hline 97. & Cyamopsis tetragonoloba (L.) Taub. & Kottavarai & Fabaceae & Herb \\
\hline 98. & Leucaena leucocephala (Lam.)de Wit & - ------------ & Fabaceae & Tree \\
\hline 99. & Mimosa pudica L. & Thottachinungi & Fabaceae & Herb \\
\hline 100. & Pithecellobium dulce (Roxb.) Benth. & Kodukkapuli & Fabaceae & Tree \\
\hline 101. & Pongamia pinnata (L.) Pierre & Pungai maram & Fabaceae & Tree \\
\hline 102. & Sesbania grandiflora (L.) Pers. & Agathi keerai & Fabaceae & Tree \\
\hline 103. & Sesbania sesban (L.) Merr. & Sittragathi & Fabaceae & Shrub \\
\hline 104. & Trigonellefoenum-graecum L. & Venthya keerai & Fabaceae & Herb \\
\hline 105. & Vigna radiata (L.) R. Wilczek & Siru pairu & Fabaceae & Herb \\
\hline 106. & Vigna unguiculata (L.) Walp. & Thata pairu & Fabaceae & Herb \\
\hline 107. & Bauhinia tomentosa L. & Iruvachi & Fabaceae & Tree \\
\hline 108. & Bauhinia variegata (L.)Benth & Semmantharai & Fabaceae & Tree \\
\hline 109. & Coleus blumi Benth & 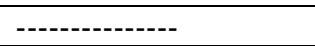 & Lamiaceae & Herb \\
\hline 110. & Mentha spicata L. & Puthina & Lamiaceae & Herb \\
\hline 111. & Ocimum tenuiflorum L. & Thulasi & Lamiaceae & Shrub \\
\hline 112. & Plectranthus amboinicus (Lour.) Spreng. & Karpooravalli elai & Lamiaceae & Herb \\
\hline 113. & Volkameria inermis L. & Shangam cooppy & Lamiaceae & Shrub \\
\hline 114. & Delonix regia (Boj. ex Hook.) Raf. & Vaagai & Leguminoceae & Tree \\
\hline 115. & Lawsonia inermis L. & Marudhani & Lythraceae & Tree \\
\hline 116. & Punica granatum L. & Maadhulai & Lythraceae & Tree \\
\hline 117. & $\begin{array}{l}\text { Michelia champaca (L.) Baill. ex } \\
\text { Pierre }\end{array}$ & Shenbaga poo & Magnoliaceae & Tree \\
\hline
\end{tabular}


International Journal of Trend in Scientific Research and Development (IJTSRD) @ www.ijtsrd.com eISSN: 2456-6470

\begin{tabular}{|c|c|c|c|c|}
\hline 118. & Abelmoschus esculentus (L.) Moench & Vendai & Malvaceae & Shrub \\
\hline 119. & Ceiba pentandra (L.) Gaertn. & $\begin{array}{l}\text { Ilavam panchu } \\
\text { maram }\end{array}$ & Malvaceae & Tree \\
\hline 120. & Guazuma ulmifolia Lam. & Thenmaram & Malvaceae & Tree \\
\hline 121. & Hibiscus rosa-sinensis L. & Chemparuthy & Malvaceae & Shrub \\
\hline 122. & Thespesia populnea (L.) Sol. Ex Correa & Poovarasu & Malvaceae & Tree \\
\hline 123. & Azadirachta indica A. Juss. & Vembu & Meliaceae & Tree \\
\hline 124. & Melia azedarach L. & Malai vempu & Meliaceae & Tree \\
\hline 125. & Albizzia lebbeck (L.) Benth & ----------- & Mimisaceae & Tree \\
\hline 126. & Moringa oleifera Lam. & Murungai & Moringaceae & Tree \\
\hline 127. & Ensete superbum Roxb. & Kal valai & Musaceae & Tree \\
\hline 128. & Musa paradisiaca L. & Vazhai & Musaceae & Tree \\
\hline 129. & Eucalyptus globus L. & Thailamaram & Myrtaceae & Tree \\
\hline 130. & Psidium guajava L. & Koiya & Myrtaceae & Tree \\
\hline 131. & Syzygium cumini (L.) Skeels & Naval maram & Myrtaceae & Tree \\
\hline 132. & Nephrolepis multiflora F.M.Jarett ex C.V.Morton & 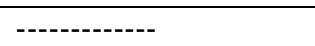 & Nephrolepidaceae & Shrub \\
\hline 133. & Bougainvillea spectabilis Willd. & Thaal poo & Nyctaginaceae & Climber \\
\hline 134. & Mirabilis jalapa L. & Anthimantharai & Nyctaginaceae & Herb \\
\hline 135. & Nymphaea alba L. & Water lily & Nymphaeaceae & Herb \\
\hline 136. & Jasminum auriculatum Vahl & Mullai & Oleaceae & Twiner \\
\hline 137. & Jasminum grandiflorum L. & Pitchi & Oleaceae & Twiner \\
\hline 138. & $\begin{array}{l}\text { Jasminum multiflorum } \\
\text { (Burm. f.) Andrews }\end{array}$ & Kasturi- mallikai & Oleaceae & Twiner \\
\hline 139. & Jasminum sambac (L.) Sol. & Kundumalli & Oleaceae & Shrub \\
\hline 140. & Nyctanthes arbor-tristis L. & Parijatham & Oleaceae & Shrub \\
\hline 141. & Spathoglottis plicata Blume & 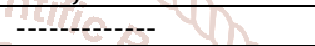 & Orchidaceae & Shrub \\
\hline 142. & Pandanus amarylifolius Roxb. & Ramba $Q$ & Pandanaceae & Tree/Shrub \\
\hline 143. & Pedalium murex L. & Aana nerunchi & Pedaliaceae & Herb \\
\hline 144. & \begin{tabular}{ll|l|l|} 
Phyllanthus acidus (L.) Skeels & 0 & 0
\end{tabular} & Pulipu nelli & Phyllanthaceae & Tree \\
\hline 145. & Phyllanthus amarus L. & Keelanelli 3 & Phyllanthaceae & Herb \\
\hline 146. & Phyllanthus emblica L. & Periya nelli & Phyllanthaceae & Tree \\
\hline 147. & Piper betle L. & Vetrilai kodi & Piperaceae & Climber \\
\hline 148. & $\begin{array}{l}\text { Russelia equisetiformis } \\
\text { Schlecht. \& Cham. }\end{array}$ & hand 90 & Plantaginaceae & Shrub \\
\hline 149. & Plumbago zeylanica L. & Chittiramoolam & Plumbaginaceae & Herb \\
\hline 150. & Bambusa vulgaris Schrad ex J.C. Wendl $\quad[S S N=24$ & Mungil & Poaceae & Tree \\
\hline 151. & Zea mays L. & Cholam & Poaceae & Herb \\
\hline 152. & Portulaca grandiflora Hook. & Pattu rose & Portulacaceae & Herb \\
\hline 153. & Ziziphus jujuba Mill. & Elandhai & Rhamnaceae & Tree \\
\hline 154. & Rosa domestica L. & Roja $>$ & Rosaceae & Shrub \\
\hline 155. & Hamelia patensJacq. & Theepputhar & Rubiaceae & Small tree \\
\hline 156. & Ixora coccinea L. & Vetchi poo & Rubiaceae & Shrub \\
\hline 157. & Knoxia hybrid L. & ----------- & Rubiaceae & Herb \\
\hline 158. & Penta lanceolata (Forssk.) Deflers & Pavazhamalli & Rubiaceae & Shrub \\
\hline 159. & Aegle marmelos L. & Vilva maram & Rutaceae & Tree \\
\hline 160. & Citrus bergamia Risso & Naarthangai & Rutaceae & Tree \\
\hline 161. & Citrus limetta Risso & Sathukudi & Rutaceae & Tree \\
\hline 162. & Citrus limon (L Burm.f.) & Elumicchai & Rutaceae & Tree \\
\hline 163. & Murraya koenigii (L.) Spreng. & Kariveppilai & Rutaceae & Tree \\
\hline 164. & Cardiospermum halicacabum L. & Mudakattan & Sapindaceae & Climber \\
\hline 165. & Manilkara zapota (L.) P. Royen & Sappota & Sapotaceae & Tree \\
\hline 166. & Mimusops elengi L. & Magizhamboo & Sapotaceae & Tree \\
\hline 167. & Capsicum annuum L. & Milagai & Solanaceae & Shrub \\
\hline 168. & Datura metel L. & Umatai & Solanaceae & Herb \\
\hline 169. & Solanum americanum Mill. & Manathakkali & Solanaceae & Herb \\
\hline 170. & Solanum lycopersicum L. & Thakkaali & Solanaceae & Shrub \\
\hline 171. & Solanum melongena L. & Katharika & Solanaceae & Shrub \\
\hline 172. & Solanum torvum Dunal & Sundaikai & Solanaceae & Shrub \\
\hline 173. & Solanum trilobatum L. & Thuthuvalai & Solanaceae & Climber \\
\hline 174. & Solanum virginianum L. & Kandankatrikai & Solanaceae & Herb \\
\hline 175. & Withania coagulans (Stocks) Dunal & Amukura & Solanaceae & Herb \\
\hline 176. & Lantana camera L. & Unni chedi & Verbenaceae & Shrub \\
\hline 177. & Tectona grandis L.f. & Tekku & Verbenaceae & Tree \\
\hline
\end{tabular}


International Journal of Trend in Scientific Research and Development (IJTSRD) @ www.ijtsrd.com eISSN: 2456-6470

\begin{tabular}{|l|l|l|l|l|}
\hline 178. & Cissus quadrangularis L. & Pirandai kodi & Vitaceae & Climber \\
\hline 179. & Vitis vinifera L. & --------- & Vitaceae & Climber \\
\hline 180. & Zamia furfuracea L. & --------- & Zamiaceae & Herb \\
\hline 181. & Curcuma longa L. & Manjal & Zingiberaceae & Herb \\
\hline 182. & Zingiber officinale Roscoe & Inji & Zingiberaceae & Herb \\
\hline
\end{tabular}

Taxonomically dicotyledons plants represent the more number of species contribute 150 species belonging to 53 families, whereas monocotyledonous plants contribute 29 species belonging to 15 families, gymnosperm presented with 2 families and 2 species and one pteridophyte species were documented in the study area.

Fabaceae (16 species and 13 Genera), Apocyanaceae ( 11 species and 10 Genera) and Solanaceae ( 9 species and 4 Genera) were the most dominant family in this home garden. Acanthaceae ( 7 speccies and 6 genera), Cucurbitaceae ( 7 species and 7 genera), Euphorbiaceae ( 7 species and 4 genera), Lamiaceae ( 5 species and 5 genera), Asteraceae ( 5 species and 5 genera), Oleaceae ( 5 species and 2 genera), Asparagaceae ( 5 species and 5 genera), Amaranthaceae ( 6 species and 5 genera), Rutaceae ( 5 species and 3 genera), Malvaceae ( 5 species and 5 genera), Arecaceae ( 5 species and 5 genera), Caesalpinaceae ( 5 species and 4 genera), Rubiaceae ( 4 species and 4 genera), Araceae ( 3 species and 3 genera), Amaryillidaceae ( 4 species and 4 genera), Myrtaceae ( 3 species and 3 genera), Costaceae ( 3 species and 2 genera), Phyllanthaceae ( 3 species and 1 genera), Anacardiaceae, Annonaceae, Apiaceae, Araceae, Cactaceae, Combretaceae, Cycadaceae, Lythraceae, Meliaceae, Musaceae, Nyctaginaceae, Plantaginaceae, Poaceae, Sapotaceae, Verbenaceae, Vitaceae and Zingiberaceae were represented by two species each, whereas Acoraceae, Agavaceae, Araucariaceae, Asphodelaceae, Balsaminaceae, Basellaceae, Bignoniaceae, Boraginaceae, Brassicaceae, Bromeliaceae, Caricaceae, Casuarinaceae, Commelinaceae, Convolvulaceae, Cupressaceae, Elaeocarpaceae, Leguminoceae, Magnoliaceae, Mimisaceae, Moringaceae, Nymphaeaceae, Orchidaceae, Palmaceae, Pandanaceae, Pedaliaceae, Piperaceae, Plumbaginaceae, Portulacaceae, Rhamnaceae, Rosaceae, Sapindaceae, Simarobauceae, Zamiaceae and Nephrolepidaceae (Pteridophytes) were mono specific. Zamiaceae and Cupressaceae families are gymnosperm. Nephrolepidaceae was presented in Pteridophytes (Table 2).

Table No. 2: Distribution of plant families in the study area

\begin{tabular}{|c|c|c|c|c|c|c|c|}
\hline S. No & Family & Genus & Species & S. No & Family & Genus & Species \\
\hline 1. & Acanthaceae & 6 & 7 & 36 & Lamiaceae & 7 & 7 \\
\hline 2. & Acoraceae & 1 & 14 & 37. & Leguminosae & 2 & 2 \\
\hline 3. & Agavaceae & 1 & terntio & 38. & Lythraceae & 2 & 2 \\
\hline 4. & Amaranthaceae & 5 & -6 & 39. & Magnoliaceae & 1 & 1 \\
\hline 5. & Amaryillidaceae & 2 & 2 & 40. & Malvaceae & 5 & 5 \\
\hline 6. & Anacardiaceae & 2 & Rea & 41. & Meliaceae & 2 & 2 \\
\hline 7. & Annonaceae & 2 & D2vel & 42. & Mimisaceae & 1 & 1 \\
\hline 8. & Apiaceae & 2 & 2 & 43. & Moringaceae & 1 & 1 \\
\hline 9. & Apocyanaceae & 8 & 1512 & 44. & Musaceae & 2 & 2 \\
\hline 10. & Araceae & 3 & 3 & 45. & Myrtaceae & 3 & 3 \\
\hline 11. & Fabaceae & 13 & 17 & 46. & Nephrolepidaceae & 1 & 1 \\
\hline 12. & Araucariaceae & 1 & 1 & 47. & Nyctaginaceae & 2 & 2 \\
\hline 13. & Arecaceae & 3 & 3 & 48. & Nymphaeaceae & 1 & 1 \\
\hline 14. & Asparagaceae & 5 & 5 & 49. & Ochidaceae & 1 & 1 \\
\hline 15. & Asphodelaceae & 1 & 1 & 50. & Oleaceae & 2 & 5 \\
\hline 16. & Asteraceae & 6 & 7 & 51. & Palmaceae & 1 & 1 \\
\hline 17. & Balsaminaceae & 1 & 1 & 52. & Pandanaceae & 1 & 1 \\
\hline 18. & Basellaceae & 1 & 1 & 53. & $\underline{\text { Pedaliaceae }}$ & 1 & 1 \\
\hline 19. & $\underline{\text { Bignoniaceae }}$ & 1 & 1 & 54. & Phyllanthaceae & 1 & 3 \\
\hline 20. & Boraginaceae & 1 & 1 & 55. & Piperaceae & 1 & 1 \\
\hline 21. & Brassicaceae & 1 & 1 & 56. & Plantaginaceae & 2 & 2 \\
\hline 22. & Bromeliaceae & 1 & 1 & 57. & Plumbaginaceae & 1 & 1 \\
\hline 23. & Cactaceae & 2 & 2 & 58. & Poaceae & 2 & 2 \\
\hline 24. & Caesalpinaceae & 2 & 2 & 59. & Portulacaceae & 1 & 1 \\
\hline 25. & Caricaceae & 1 & 1 & 60. & Rhamnaceae & 1 & 1 \\
\hline 26. & Casuarinaceae & 1 & 1 & 61. & Rosaceae & 1 & 1 \\
\hline 27. & Combretaceae & 1 & 2 & 62. & Rubiaceae & 4 & 4 \\
\hline 28. & Commelinaceae & 1 & 1 & 63. & Rutaceae & 3 & 5 \\
\hline 29. & Convolvulaceae & 1 & 1 & 64. & Sapindaceae & 1 & 1 \\
\hline 30. & $\underline{\text { Costaceae }}$ & 2 & 3 & 65. & Sapotaceae & 2 & 2 \\
\hline 31. & $\underline{\text { Cucurbitaceae }}$ & 7 & 7 & 66. & Simarobauceae & 1 & 1 \\
\hline 32. & $\underline{\text { Cupressaceae }}$ & 1 & 1 & 67. & Solanaceae & 4 & 10 \\
\hline 33. & Cycadaceae & 1 & 2 & 68. & Verbenaceae & 1 & 1 \\
\hline 34. & Elaeocarpaceae & 1 & 1 & 69. & Vitaceae & 2 & 2 \\
\hline 35. & Euphobiaceae & 5 & 7 & 70. & Zamiaceae & 1 & 1 \\
\hline & & & & 71. & Zingiberaceae & 2 & 2 \\
\hline
\end{tabular}


Most plant species of the study area are of considerable ecological and economic importance, useful as bio resources to wild fauna and human beings. Of the total 182 wild/naturalized plant species, most are useful as medicinal plants, and others are valuable as edible fruits, timbers, fuel wood, etc. Although food production is recognized as a basic function of home gardens ${ }^{2}$ ,the motivation for home gardening is not always for mainly food production. With the recent studies on home gardens in Benin $^{20}$, they revealed high prevalence of food and medicinal plants in gardens, confirming the importance of food production in gardening, and evidencing the key importance of medicinal plant in gardening systems in Benin.

Home gardens with primarily for both food and medicinal purposes and with more functions (ornamental, protection/delimitation, and miscellaneous purposes) were found everywhere but most garden with high prevalence of ornamental plant species were also mostly found in these regions under the westernization influence. The ornamental quality of Tilzapotla's home gardens differed from that in other regions; these home gardens were mainly used for food security, to improve families' nutrition, and for economic growth in some cases ${ }^{21}$. Nevertheless, there are newly emerging positive trends in home gardening, which encourage people to maintain biodiversity in rural and urban gardens.

In developing countries the nutritional value of local, neglected horticultural species has been assessed and their cultivation in family gardens promoted to guarantee the intake of vitamins and micro-nutrients ${ }^{22}$.In high-income countries the growing demand for healthier life styles and closer connection with nature has driven a renewed interest towards sustainable agricultural systems and "traditional" food products, capable of connecting consumers to the natural and cultural heritage of a community or a geographical region. Many urban citizens of the developed world have taken up some form of self-production of food in their terraces, roofs, gardens or courtyards as well as in communal areas shared among neighbours ${ }^{23}$.

For all the enumerated wild and naturalized plant species, information such as botanical name, vernacular name, family and habit are provided and plant species in the checklist and their photographs are showed in (Fig3).

Figure Ho 3. Selected Plants of Home Genden

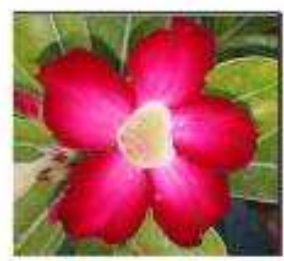

Adonium obescim Forssk.

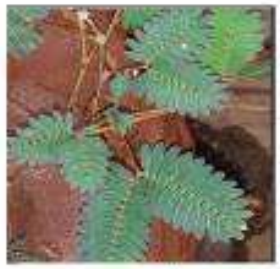

Mimosa purtica L

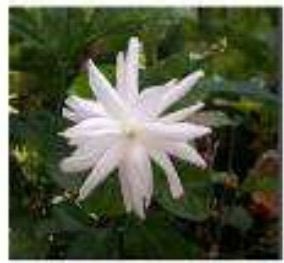

Jasminum officintale L

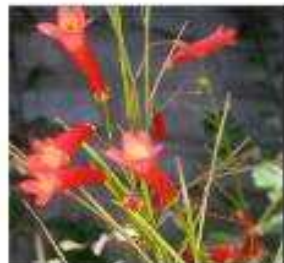

Hamelia pafens Jaco.

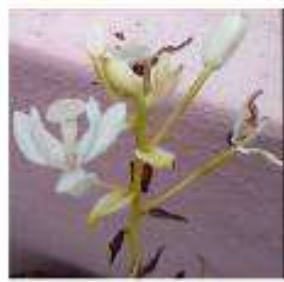

Spathogiottis plicata Btume

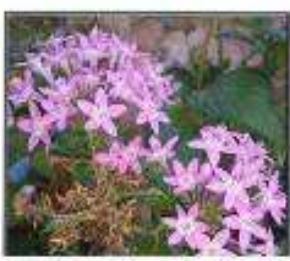

Priaxia fybonido L.

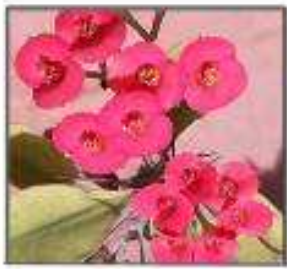

Euphorbà militi Dass Moul.

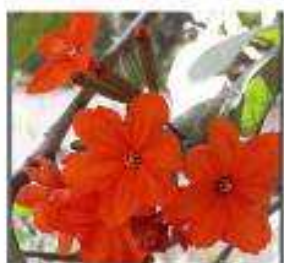

Cordia-sefestans L

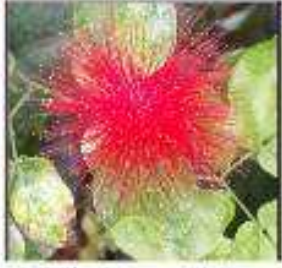

Combretum constrictum Benthis Lawson.

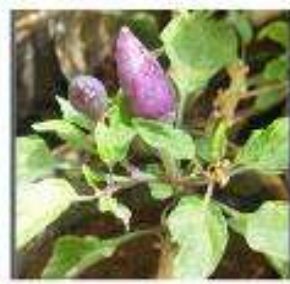

Capsicum annuam L.

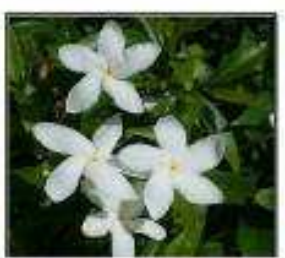

Tabenaemontana olvariçata R. Br, ex foem \&s Schult.

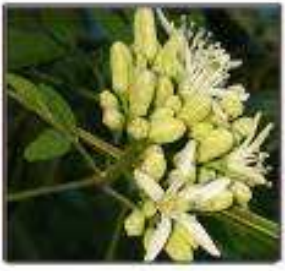

Murraya kounighi fL.) Sprengo.

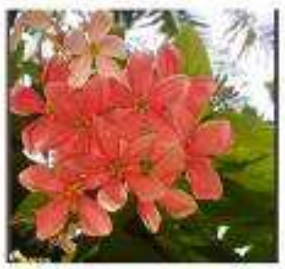

Cursquaìs indicat $\mathrm{L}$.

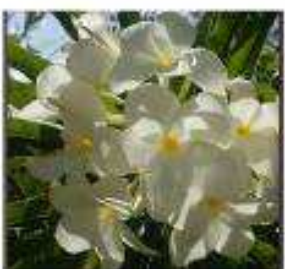

Phineria cubrs L

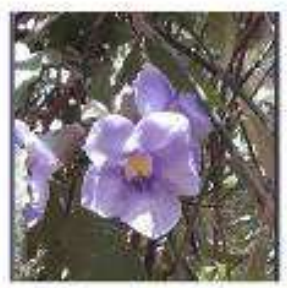

Thumbergis grandiffora Roxb.

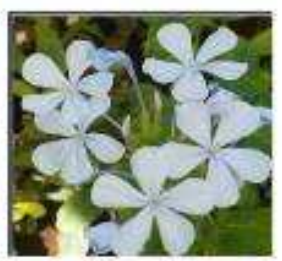

Plambago zeylarica L.

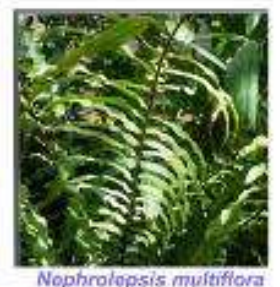

(Sw.) Schot..

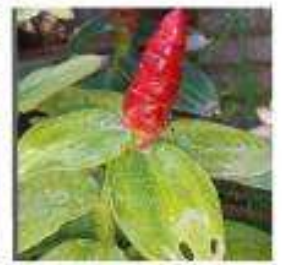

Costus wooosonil Jacq.

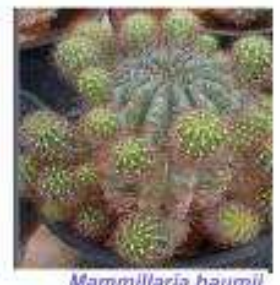

Mammillaria bavimi Hoedeker.

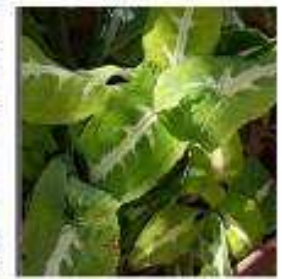

Caladium biocofar Vont. 


\section{CONCLUSION}

In this study, we observed totally of 182 plant species belonging to 159 genera and 71 families from the home gardens of Palayamkottai, Tirunelveli District. Among them, 60 species (32\%) were trees, 39 species $(21 \%)$ were shrubs, 61 species (33\%) were herbs, 18 species (10\%) were climbers and 7 species (4\%) were twiners. Home gardens provide good economic and social conditions for outstanding production. They are an important production system of food, medicine and other essential products. It also provides environments in which part of the genetic diversity for many crops species can be maintained. In conclusion, home gardens play a major role in the production of food, job opportunities, crop improvement, development, maintenance of the green nature and so on.

\section{REFERENCE}

[1] Fernandes ECM and Nair PKR. An evalution of the structure and function of tropical home gardens. Agricultural Systems.1986; 21: 279-310.

[2] Kumar BM and Nair PR. 2004. The enigma of tropical home gardens. Agrofor Syst. 2004; 61(1-3): 135-52.

[3] Gibbs L, Staiger PK Townsend M etal. Methodology for the evaluation of the Stephanie Alexander kitchen garden program. Health Promotion Journal of Australia, 2013; 24(1): 32-43.

[4] Taylor JR and Lovell ST. Urban home food gardens in the global north: research traditions and future directions. Agric Hum Values. 2014; 31(2):285-305.

[5] Zainuddin Z and Mercer D. Domestic residential garden food production in Melbourne, Australia: a fine-grained analysis and pilot study. Aust Geogr, 2014; 45(4):46584.

[6] Calvet-Mir L, Gómez-Baggethun E and Reyes-García V. Beyond food production: ecosystem services provided by home gardens. A case study in Vall Fosca, Catalan Pyrenees, Northeastern Spain. Ecol Econ, 2012; 74: 153-60.

[7] Laura CM, Hug M, Daniel CM et al. Home garden ecosystem services valuation through a gender lens: a case study in the Catalan Pyrenees. Sustainability. 2016; 8(8):718.

[8] Serranoa V, Onaindiab M, Josu et al. Plant diversity and ecosystem services in Amazonian home gardens of Ecuador. Agric Ecosyst Environ. 2016; 225: 116-25.

[9] Clarke LW, Li LG and Yu DJZ. Drives of plant biodiversity and ecosystem service production in home gardens across the Beijing municipality of China. Urban ecosystems. 2014; 17(3):741-60.

[10] Mohri H, Lahoti S, Saito O. Assessment of ecosystem services in homegarden systems in Indonesia, Sri Lanka, and Vietnam. Ecosystem Services. 2013; 5: 124136.

[11] Salako VK, Fandohan B, Kassa, B et al. Home gardens: an assessment of their biodiversity and potential contribution to conservation of threatened species and crop wild relatives in Benin. Genet Resour Crop Evol. 2014; 61(2):313-30.
[12] Idohou R, Fandohan B, Salako VK. Biodiversity conservation in home gardens: traditional knowledge, use patterns and implications for management. International Journal of Biodiversity Science, Ecosystem Services \& Management, 2014; 10(2): 89100.

[13] Cruz-Garcia and Struik PC. Spatial and seasonal diversity of wild food plants in home gardens of northeast Thailand1. Econ Bot. 2015; 69(2): 99-113.

[14] Boege E. El Patrimonio Biocultural de los Pueblos Indígenas de México: Hacia la Conservación in situ de la Biodiversidad y Agrodiversidad en los Territorios Indígenas. Instituto Nacional de Antropología e Historia. México, D.F. 2010.

[15] Toledo V, Ortiz-Espejel B, Cortés L et al. The multiple uses of tropical forests by indigenous peoples in Mexico: A case of adaptive management. Conservation Ecology. 2003; 7: 9.

[16] Blanckaert I, Swennen R, Paredes-Flores M et al. Floristic composition, plant uses and management practices in homegardens of San Rafael Coxcatlán, Valley of Tehuacán-Cuicatlán, Mexico. Journal of Arid Environments. 2004; 57: 179-202.

[17] Cano-Ramírez M, De la Tejera-Hernández B, Casas A, et al. Migración rural y huertos familiares en una comunidad indígena del centro de México. Botanical Sciences, 2012;90: 287-304.

[18] Neulinger K, Vogl CR and Alayón-Gamboa JA. Plant species and their uses in home gardens of migrant Maya and mestizo smallholder farmers in Calakmul, Campeche, Mexico. Journal of Ethnobiology. 2013;33:105-124.

[19] Srithi K, Trisonthi C, Wangpakapattanawong $\mathrm{P}$ et al. Plant diversity in Hmong and Mien home gardens in northern Thailand", Economic Botany, 2012; 66: 192206.

[20] Rodrigue I, Belarmain F, Valère KS. et al. Biodiversity conservation in home gardens: traditional knowledge, use patterns and implications for management. International Journal of Biodiversity Science, Ecosystem Services \& Management. 2014; 10(2): 89100.

[21] Casas A, Vázquez MC, Viveros JL et al. Plant management among the Náhua and the Mixtec in the Balsas River Basin, Mexico: An etnobotanical approach to the study of plant domestication. Human Ecology. 1996; 24:455-478.

[22] Odhav B, Beekrum S and Akula U. Preliminary assessment of nutritional value of traditional leafy vegetables in Kwazulu Natal, South Africa. Journal of Food Composition and Analysis. 2007; 20: 430-435.

[23] Bhatt V and Farah L. Urban design for food-security: thinking globally, designing locally. In: Proceedings of the second international conference on landscape and urban horticulture. Department of Agro Environmental Science and Technology (DSTA), Faculty of Agriculture, University of Bologna, Italy. 2009; 40. 